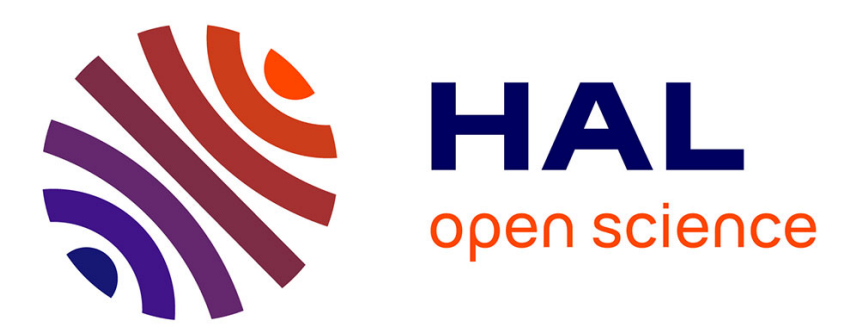

\title{
Numerical study of convection induced by evaporation in cylindrical geometry
}

Benoît Trouette, Eric Chénier, C. Delcarte, B. Guerrier

\section{To cite this version:}

Benoît Trouette, Eric Chénier, C. Delcarte, B. Guerrier. Numerical study of convection induced by evaporation in cylindrical geometry. The European Physical Journal. Special Topics, 2011, 192, pp.83-93. 10.1140/epjst/e2011-01362-x . hal-00691214

\section{HAL Id: hal-00691214 \\ https://hal.science/hal-00691214}

Submitted on 25 Apr 2012

HAL is a multi-disciplinary open access archive for the deposit and dissemination of scientific research documents, whether they are published or not. The documents may come from teaching and research institutions in France or abroad, or from public or private research centers.
L'archive ouverte pluridisciplinaire HAL, est destinée au dépôt et à la diffusion de documents scientifiques de niveau recherche, publiés ou non, émanant des établissements d'enseignement et de recherche français ou étrangers, des laboratoires publics ou privés. 


\title{
Numerical study of convection induced by evaporation in cylindrical geometry
}

\author{
Benoît Trouette $^{1}$, Eric Chénier ${ }^{2}$ a , Claudine Delcarte ${ }^{1}$, and Béatrice Guerrier ${ }^{3}$ \\ 1 Univ. Paris-Sud XI, LIMSI-CNRS, B.P. 133, 91403 Orsay Cedex, FRANCE \\ 2 Univ. Paris-Est, Lab. MSME, UMR 8208 CNRS, 77454 Marne-la-Vallée, FRANCE \\ 3 UPMC Univ. Paris VI, Univ. Paris-Sud, CNRS, lab. FAST, Bât. 502, 91405 Orsay Cedex, FRANCE
}

\begin{abstract}
Numerical simulations of convection induced by solvent evaporation during the drying of a polymer solution are considered. This paper focuses on the transient thermal regime occurring at the beginning of the drying and transient solutal effects are not taken into account. The onset of convection (BénardMarangoni and Rayleigh-Bénard) is studied for a large range of initial thicknesses and viscosities. Several stochastic models are compared to analyze the influence of the perturbation description on the transition thresholds. Two-dimensional $(2 D)$ and three-dimensinal $(3 D)$ models are shown to give close results. The $3 D$ model is used to characterize the pattern evolution during the drying. In the case of surface tension driven convection, a method is developed to describe the cells morphology and their time evolution.
\end{abstract}

\section{Introduction}

The drying of binary fluid films including one volatile component appears in many industrial processes such as painting, inking or packaging. When drying a polymer/solvent solution, the vaporization of the solvent induces a temperature decrease at the free surface due to the latent heat transfer and the increase of the solute concentration. The resulting thermal and mass gradients create volume (buoyancy) and surface (capillary) forces that can generate a flow into the bulk [1-9]. One important point is that the flow is transient in most configurations. Indeed starting from a fluid at rest at ambient temperature, a transient diffusive regime takes place as soon as evaporation begins. Over time, the heat and mass transfers evolve and can generate convection. Sometime after, the fluid motion fades away due to thermal or solutal gradients changes and/or thickness reduction and viscosity increase. At the end, transfers are governed by diffusion again.

The numerical approach presented here follows an experimental study dealing with drying of polymer solutions. The development and evolution of convective patterns induced by solvent evaporation in a Polyisobutylene/toluene solution have been experimentally studied for a large range of initial thicknesses and viscosities [10]. In this paper we focus on the thermal transient regime that concerns the beginning of the drying only; solutal effects are disregarded. $2 D$ numerical simulations [11] and linear stability analysis [12] have been performed for the transient thermal regime. Critical Marangoni and Rayleigh numbers have been determined as a function of the experimental control parameters. As pointed out in these previous works, it is more proper to view the results as the definition of a transition region rather than well defined threshold values between stable and unstable domains. Indeed, in transient problems, the thresholds depend on the criterion used to characterize significant convection, and also on

\footnotetext{
a e-mail: eric.chenier@univ-paris-est.fr
} 
the initial perturbation. It is then important to check that this transition region is thin and slightly depends on the perturbation characteristics (structure and amplitude). In the previous $2 D$ numerical study [11], the initial condition consisted of a random vector field defined on collocation nodes. One of the purposes of this paper is to introduce more sophisticated models, closer to realistic physical behaviors, by using stochastic approaches and by taking into account spatial correlations for disturbances. The second point developed in this study is the extension of the $2 D$ thermal model to the $3 D$ configuration. Convection/diffusion threshold values are discussed and compared with $2 D$ results. A particular attention is paid to flow structures at the beginning of the drying that cannot be described in $2 D$ simulations.

\section{Physical and mathematical model}

The numerical simulations refer to experiments of polymer solutions drying [10]. Details on the approximations that have been considered for the thermal transient regime occurring at the beginning of the drying can be found in [11] and [12]. They are briefly recalled below.

A cylindrical container of radius $R$, with adiabatic solid surfaces, is filled with a Polyisobutylene/toluene solution over a thickness $e$ (Fig. 1). Experimental visualizations by means

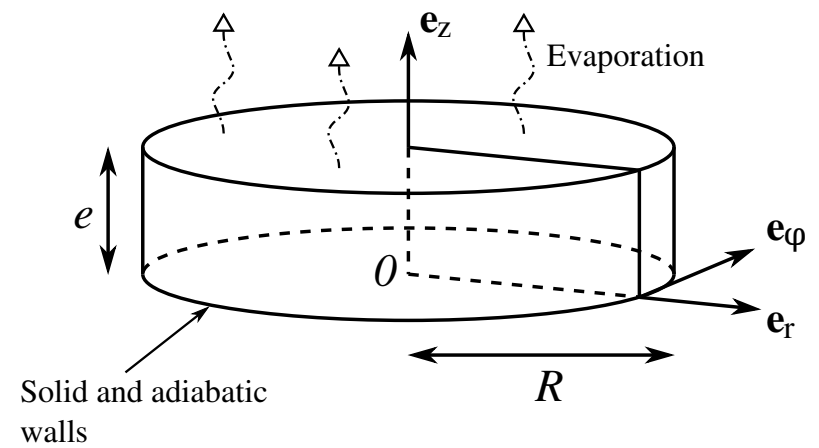

Fig. 1. Scheme of the configuration

of I.R. camera show some convective instabilities occuring on short periods of the order of some thermal diffusion times, this last time scale being $10^{-3}$ smaller than the solutal diffusion time scale. During this thermal regime, the solutal effects and decrease of the solution thickness are neglected. The mass fraction of the solvent is then assumed constant and the viscosity used in the simulations corresponds to the initial solute concentration. The two control parameters chosen in the experiments were the initial thickness $(0.3 \mathrm{~mm} \leq e \leq 14.3 \mathrm{~mm})$ and the initial viscosity through the initial polymer mass fraction $\left(0 \% \leq \omega_{p} \leq 1 \overline{5} \%\right.$ corresponding to $5.5 \times 10^{-4}$ Pa.s $\leq \mu \leq 2.4$ Pa.s $)$. Given the physical properties of the solution and the geometric configuration, it can be shown that the instability mode due to the coupling between the Marangoni effect and the deflection of the free surface can be neglected [12]. Moreover, a non-zero base evaporation rate stabilizes the interface against deformation due to vapor dynamics [8]. The modelisation of the gas phase is here ignored and the free surface deformation is disregarded. The interface between the solution and surounding gas is then considered as flat. The fluid is assumed Newtonian. Except for the viscosity, physical properties are those of the pure solvent. They are kept constant except for both the density in the buoyant term (Boussinesq approximation) and the surface tension in the capillary stress which depend linearly on the temperature. The thermal regime is thus governed by the Navier-Stokes and energy equations 
in dimensionless form:

$$
\left\{\begin{array}{l}
\frac{\partial \boldsymbol{u}}{\partial t}+(\boldsymbol{u} \cdot \boldsymbol{\nabla}) \boldsymbol{u}=-\nabla p+\operatorname{Pr} \Delta \boldsymbol{u}+\operatorname{RaPr} \Theta \mathbf{1}_{z} \\
\frac{\partial \Theta}{\partial t}+(\boldsymbol{u} \cdot \boldsymbol{\nabla}) \Theta=\Delta \Theta \\
\boldsymbol{\nabla} \cdot \boldsymbol{u}=0
\end{array}\right.
$$

where $\boldsymbol{u}=(\boldsymbol{u} \cdot \boldsymbol{\tau}) \boldsymbol{\tau}+\left(\boldsymbol{u} \cdot \mathbf{1}_{z}\right) \mathbf{1}_{z}, p$ and $\Theta$ respectively denote the dimensionless velocity, pressure and temperature fields; $\mathbf{1}_{z}$ stands for the upward unit vector normal to the free surface and $\boldsymbol{\tau}$ is unitary, such that $\boldsymbol{\tau} \cdot \mathbf{1}_{z}=0$. The position vector $\boldsymbol{x}$ writes in cylindrical coordinates $\left(\mathbf{1}_{r}, \mathbf{1}_{\phi}, \mathbf{1}_{z}\right)$, $\boldsymbol{x}=(r, \phi, z)$ and in $2 D$-Cartesian coordinates $\left(\mathbf{1}_{x}, \mathbf{1}_{z}\right), \boldsymbol{x}=(x, z)$; the velocities are expressed by $\boldsymbol{u}=u \mathbf{1}_{r}+v \mathbf{1}_{\phi}+w \mathbf{1}_{z}$ and $\boldsymbol{u}=u \mathbf{1}_{x}+w \mathbf{1}_{z}$, respectively. The length, velocity, pressure and time are scaled by the fluid thickness $e$, the thermal diffusion velocity $\alpha / e$, the dynamical pressure $\rho \alpha^{2} / e^{2}$ and the thermal diffusion time $e^{2} / \alpha$, where $\alpha$ and $\rho$ are the thermal diffusivity and the density of the solution. The reduced temperature is defined by $\Theta=\left(T-T_{0}\right) / \Delta T_{s}$ where $\Delta T_{s}$ is the difference between the initial temperature $T_{0}$ and the steady and uniform temperature at the end of the transient thermal regime. The temperature scale results from the balance, at the free surface, between the heat flux lost by evaporation, the convective heat transfer with the surrounding gas and the conduction in the liquid film. A one-sided model is used and heat transfer with the surroundings is taken into account by a constant and uniform convective coefficient $h$. Assuming small temperature variations, the evaporation flux can be linearized, given [11]:

$$
-\left.\lambda \frac{\partial T}{\partial z}\right|_{(z=e)}=h\left(T(z=e)-T_{0}\right)+L\left(\Phi\left(T_{0}\right)+\left.\frac{\partial \Phi}{\partial T}\right|_{T_{0}}\left(T(z=e)-T_{0}\right)\right)
$$

with $\lambda$ the thermal conductivity, $\Phi\left(T_{0}\right)$ the evaporative heat flux at $T_{0}, L$ the latent heat. Setting $H=h+\left.L \frac{\partial \Phi}{\partial T}\right|_{T_{0}}$, the global exchange coefficient, we obtain

$$
-\left.\lambda \frac{\partial T}{\partial z}\right|_{(z=e)}=H\left(T(z=e)-T_{0}\right)+L \Phi\left(T_{0}\right)
$$

At the end of the transient thermal regime, the temperature becomes steady and uniform again: the heat flux gained by convective transfer with the surroundings balances exactly the heat flux lost by evaporation. The temperature difference between the liquid and the surroundings is then $\Delta T_{s}=\frac{L \Phi\left(T_{0}\right)}{H}$. In cylindrical coordinates, the dimensionless boundary conditions write:

$$
\begin{aligned}
& \text { at } r=A \text { or } z=0 \quad \begin{cases}\boldsymbol{u}=\mathbf{0} & \text { (no slip) } \\
\frac{\partial \Theta}{\partial \boldsymbol{n}}=0 & \text { (adiabatic surfaces) }\end{cases} \\
& \text { and at } z=1 \begin{cases}\frac{\partial(\boldsymbol{u} \cdot \boldsymbol{\tau})}{\partial z}=-\mathrm{Ma} \frac{\partial \Theta}{\partial \tau} & \text { (thermocapillary stress) } \\
w=0 \quad(\mathrm{cf} .[12]) & \\
-\frac{\partial \Theta}{\partial z}=\operatorname{Bi}(\Theta+1) & \text { (energy balance) }\end{cases}
\end{aligned}
$$

The dimensionless parameters $A=R / e, \operatorname{Pr}=\nu / \alpha, \operatorname{Ra}=g \beta \Delta T e^{3} / \mu \alpha$, Ma $=-\frac{\partial \gamma}{\partial T} e \Delta T / \mu \alpha$ and $\mathrm{Bi}=H / \alpha$ are the aspect ratio of the liquid film and the Prandtl, Rayleigh, Marangoni and Biot numbers, $\mu, \nu, \gamma$ being the dynamic and kinematics viscosities and the surface tension coefficient respectively. The dimensionless parameters $\mathrm{Pr}, \mathrm{Ra}$, Ma and $\mathrm{Bi}$ depend on two physical control parameters used in experiments, namely the initial fluid thickness and the initial viscosity (which is a function of the polymer mass fraction [10]); the relations between control 


\begin{tabular}{|c|c|c|c|}
\hline $\mathrm{Ma}$ & $\mathrm{Ra}$ & $\mathrm{Pr}$ & $\mathrm{Bi}$ \\
\hline $5850 e / \mu$ & $451 e^{3} / \mu$ & $12 \mu$ & $0.2 e$ \\
\hline
\end{tabular}

Table 1. Relations between the dimensionless parameters and the thicknesses $([e]=\mathrm{mm})$ and viscosities $([\mu]=\mathrm{mPa} . \mathrm{s})$

parameters and dimensionless numbers are given in Table 1.

For the $3 D$ configuration, a pseudo-spectral method has been developed to solve the equations (1) with boundary conditions (2) in cylindrical coordinates $(r, \phi, z)$ [13]. The solution in the azimuthal direction has been expanded in Fourier series with 128 complex conjugate modes. The evaluations of the radial and axial space derivatives are based on Chebyshev collocation method with typically 181 Gauss-Radau and 20 Gauss-Lobatto points. Convective and advective terms are handled with a second order Adams Bashforth scheme, the diffusion contributions being implicitly treated. Velocity and pressure decoupling is achieved by projection algorithm. Computations are initialized with the fluid at rest and with small disturbances added to the uniform temperature field (cf. next section).

To study the sensitivity of the transient flow to the initial disturbances, a $2 D$ model has also been used. The equations have been solved, in Cartesian geometry $(x, z)$, using finite volume discretization with second order space approximations. The rectangular liquid layer length $2 \mathrm{~A}$ corresponds to the diameter of the container. The time discretization scheme and the pressurevelocity decoupling are similar to those used in $3 D$ simulations.

\section{Uncertainty model for the initial thermal condition}

The stochastic approach proposed in this paper aims to introduce relevant uncertainties in the model in order to better take into account experimental conditions.

As previously said, the fluid is initially at rest, $\mathbf{u}=\mathbf{0}$, and perturbations are applied on the initial temperature only. The mean value of the resulting field is equal to the ambient temperature, i.e. zero in reduced variables. Using the subscript $\xi$ to denote stochastic variables, the initial temperature field $\Theta_{\xi}(\boldsymbol{x}, t=0)$ writes:

$$
\Theta_{\xi}(\boldsymbol{x}, t=0)=0+\theta_{\xi}(\boldsymbol{x})
$$

The random variables $\xi$ are here provided by a uniform and centered probability density function $p(\xi)=\sqrt{3} / 6$ on $[-\sqrt{3} ; \sqrt{3}]$ such that the mean value $E(\xi)=\int_{\mathbb{R}} \xi p(\xi) d \xi=0$ and the variance $E\left(\xi^{2}\right)=\int_{\mathbb{R}} \xi^{2} p(\xi) d \xi=1$. The evolutions of the velocity and temperature are then analyzed by means of mean values and standard errors. These statistical quantities are computed by a Monte Carlo method which necessitates numerous deterministic simulations. The random samples are obtained by a Twister Mersenne algorithm [14]. Several stochastic models of $\theta_{\xi}(\boldsymbol{x})$ have been compared. The first model hes been used for both $2 D$ and $3 D$ simulations and the others only in the $2 D$ approach in Cartesian geometries, in order to limit computational time. For simplicity, equations are only given for the $2 D$-Cartesian configuration, with $-A \leq x \leq A$ and $0 \leq z \leq 1$.

1. The simplest idea consists in taking into account the uncertainties directly in the numerical scheme. To do this, we define the discrete initial condition so that a random value, defined by the probability law $p(\xi)$, is accociated to each collocation node for the temperature. Using the finite volume method with $N_{x}+1$ discrete $x$-coordinates $x_{i}=\left(2 i-N_{x}\right) / N_{x} \times A, i=0, \cdots, N_{x}$ and $N_{z}+1$ discrete $z$-coordinates $z_{j}=j / N_{z}, j=0, \cdots, N_{z}$, and two independent families of random variables $\left\{\xi_{i}, i=0, \cdots, N_{x}\right\}$ and $\left\{\xi_{j}, j=0, \cdots, N_{z}\right\}$ defined by $p(\xi)$, the stochastic initial condition writes:

$$
\theta_{\xi}(x, z)=\frac{a \sqrt{3}}{6} \sum_{i=0}^{N_{x}-1} \sum_{j=0}^{N_{z}-1} H\left(x-x_{i}\right) H\left(x_{i+1}-x\right) H\left(z-z_{j}\right) H\left(z_{j+1}-z\right) \xi_{i} \xi_{j}
$$


with $H(x)$ the Heaviside function and $a$ the maximum magnitude of the disturbance. Such an approach is very easy to implement but may be questionable because of the non regularity of the initial thermal condition. Indeed spectral methods, and in a smaller extent finite volume methods, require regular solutions.

The gradients of the initial temperature diverge as a function of the mesh size, so that convergence analysis cannot be easily performed. Despite this point, this simple stochastic approach has been used for all computations performed with the spectral $3 D$ code.

2. In order to guarantee the regularity of the initial thermal condition, we assume that disturbances are spatially correlated. This assumption is also physically relevant because the smallest patterns are necessarily driven by diffusion scales. For the sake of simplicity and considering the lack of information about physical disturbances, we suppose that the random thermal initial condition only depends on the horizontal coordinate $x$ and is invariant in the $z$-direction. The stochastic initial condition is then expressed by a Karhunen-Loève decomposition [15]:

$$
\theta_{\xi}(x, z)=\sum_{m=0}^{\infty} \sqrt{\beta_{m}} \xi_{m} \phi_{m}(x)
$$

where $\left\{\phi_{m}(x)\right\}$ is a Hilbert base in $L^{2}([-A ; A]), \beta_{m}>0$ such that $\beta_{i+1} \leq \beta_{i}$ and $\lim _{n \rightarrow \infty} \beta_{n}=$ 0 , and $\left\{\xi_{m}\right\}$ is a family of random values defined by the probability law $p(\xi)$. The autocorrelation function $R\left(x, x^{\prime}\right)$ was chosen to enable analytical calculations:

$$
R\left(x, x^{\prime}\right)=R_{0} \exp \left(\frac{-\left|x-x^{\prime}\right|}{\lambda}\right)
$$

with $\lambda$ the correlation length [16]. It can be shown that:

$$
\left\{\begin{array}{l}
\phi_{n}(x)=A_{n} \cos \left(\omega_{n} x+\chi_{n}\right) \\
A_{n}=\sqrt{\frac{2 \omega_{n}}{2 A \omega_{n}+\sin \left(2 A \omega_{n}\right) \cos \left(2 A \omega_{n}+2 \chi_{n}\right)}} \\
\beta_{n}=\frac{2 \lambda R_{0}}{1+\lambda^{2} \omega_{n}^{2}} \\
\chi_{n}=-A \omega_{n}+\frac{n \pi}{2} \\
\tan \left(A \omega_{n}-\frac{n \pi}{2}\right)=\frac{1}{\omega_{n} \lambda}
\end{array}\right.
$$

3. The third stochastic model uses an uncorrelated disturbance similar to the first model, but only in $x$-direction. Like in the second model the random thermal initial condition is therefore invariant in $z$-direction.

$$
\theta_{\xi}(x, z)=\frac{a \sqrt{3}}{6} \sum_{i=0}^{N_{x}-1} H\left(x-x_{i}\right) H\left(x_{i+1}-x\right) \xi_{i}
$$

In order to introduce the same thermal energy $\left(L_{2}\right.$-norm $)$ at the initial time, the intensity of the auto-correlation function fulfills the equality $R_{0}=a^{2} / 12$.

\section{Results}

\subsection{Onset of convection}

The results presented in this paragraph are obtained for $e=1 \mathrm{~mm}$ and $\mu=3 \mathrm{mPa} . \mathrm{s}$ and with $A=10$. Statistics are achieved with 100 and 200 random shots in $3 D$ and $2 D$, respectively.

Fig. 2 shows the transient behavior of the fluid and the comparison between the different stochastic models. Filled symbols and crosses stand for $2 D$ - and $3 D$-simulations, respectively; 


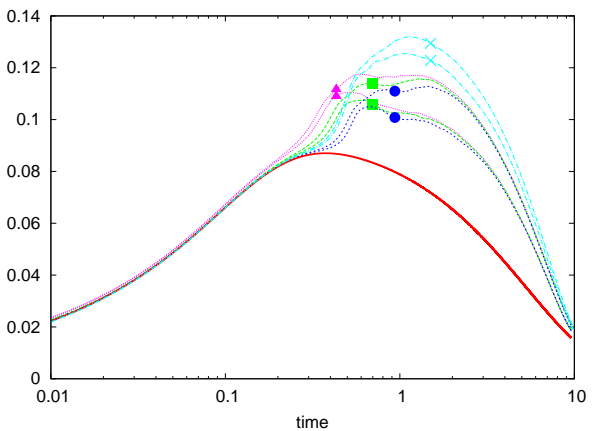

(a) Temperature difference $\Delta \Theta_{\max }$ $\max _{x}(\Theta(x, z=0, t))-\min _{x}(\Theta(x, z=1, t))$

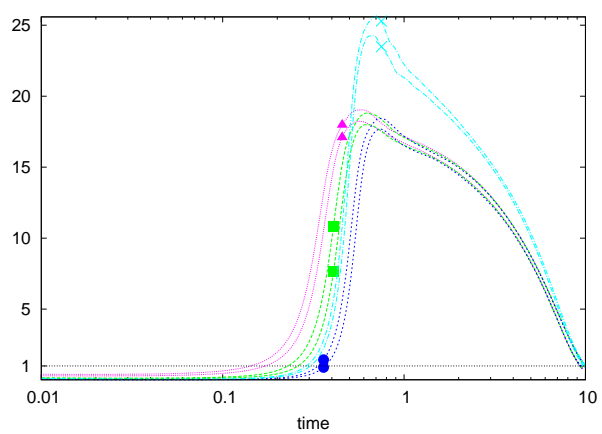

$=$ (b) Péclet number value $\mathrm{Pe}=\max _{\boldsymbol{x}}\|\boldsymbol{u}(\boldsymbol{x}, t)\|$

Fig. 2. Transient evolutions for $e=1 \mathrm{~mm}, \mu=3 \mathrm{mPa} . \mathrm{s}$ and $A=10$. The full line corresponds to the diffusion case $(\mathrm{Ra}=\mathrm{Ma}=0)$. Crosses stand for $3 D$-results with $A=10$. Filled circles (Eq. (3)), triangles (Eqs. (4) and (5) with $\lambda=1$ ) and squares (Eq. (6)) are $2 D$-results. For each symbol set, the area between the two curves corresponds to the uncertainty domain based on the mean value, plus and minus the standard error.

curves with the same symbols correspond to mean value plus or minus standard error. Fig. 2(a) presents $\Delta \Theta_{\max }$, the maxima of the temperature difference between the bottom and top of the fluid layer as a function of time. It must be emphasized that, when convection is on, these maxima are larger than in the pure diffusive regime (full line curve). This is linked to local effects. Indeed, once the flow is convective, the vertical thermal gradient at the free surface decreases in the regions where the fluid dives into the core bulk. The conduction being reduced at the interface (cf. Eq. (2)), the temperature at the free surface decreases due to the heat lost by evaporation (cf. negative values at $z=1$ in Fig. 3). In contrast at the bottom, the temperature is larger than in the diffusion case when the fluid flows upward (cf. positive values at $z=0$ in Fig. 3). Consequently, the maximum temperature difference between the bottom

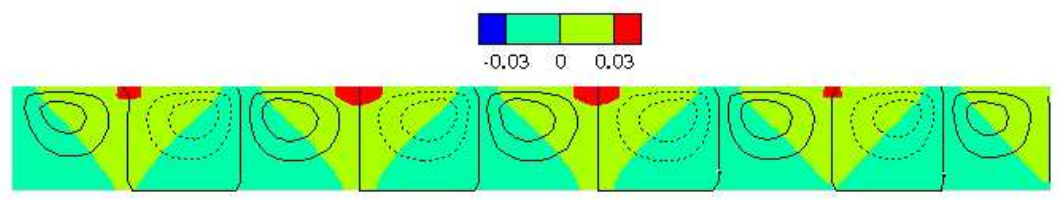

Fig. 3. Difference between the convective and conductive thermal fields and equally spaced streamlines, for $e=1 \mathrm{~mm}, \mu=3 \mathrm{mPa} . \mathrm{s},-10 \leq x \leq 0$ and $t=1$

and the interface increases beyond the diffusive value. This effect is more pronounced in the $3 D$ model since the Peclet number is about $40 \%$ higher than in the $2 D$ case. However the mean value of the temperature difference is always smaller in the convective regime than in the diffusive regime, as expected. The curves depart from the diffusive case when the convection becomes significant $(0.1<t<0.3)$. These times correspond to a Peclet number close to one, i.e. when the thermal diffusion velocity and the maximum of the velocity are of the same order (Fig. 2(b)).

Several conclusions can be drawn from these results. Whereas the mean initial thermal energy was the same in the different stochastic models, convection arises earlier with correlated disturbances because the correlation length, $\lambda=1$, is close to the natural wavelength of the convective cells (cf. Fig. 3). Disturbances based on uncorrelated random values (Eq. (3)) are 
the less effective. However the use of the three stochastic models does not affect significantly the transient flow and heat transfer, what is in good agreement with [16] and justifies the use of the simple first stochastic approach. $2 D$ and $3 D$ simulations give rather close results. Although the maximum value of the Peclet number is $40 \%$ higher in the $3 D$ case, the departure from the diffusive regime occurs more or less at the same time.

In a second step the influence of the perturbation amplitude on the onset of convention is studied. To characterize the presence of observable convection, a criterion based on the thermal Peclet number is chosen. Convection will be considered as significant if, when the system is submitted to an initial perturbation, there is a time $t$ at which the perturbation is significantly amplified, i.e. such as $\mathrm{Pe}=\max _{\boldsymbol{x}}|\boldsymbol{u}(\boldsymbol{x}, t)|>1$. Results are presented in the viscosity-thickness map (Fig. 4), for the $3 D$ model and for perturbation amplitudes varying on several orders of magnitude, $\sqrt{3} \cdot 10^{-9} \leq a \leq \sqrt{3} \cdot 10^{-3}$ (cf. Eq. (3)). As a comparison the thresholds computed with the $2 D$ model by Touazi et al. [11] are also displayed. As is made clear in the figure, on

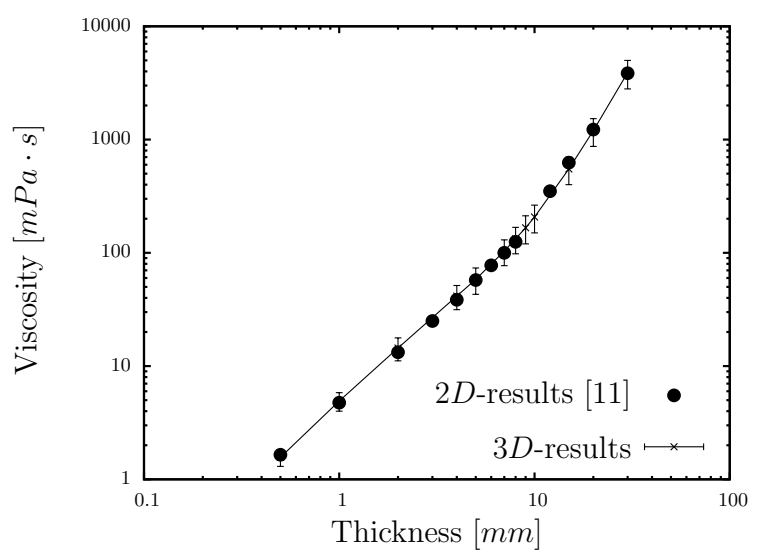

Fig. 4. Diffusion/convection transition as a function of the liquid thickness and viscosity, $A=10$. In the $3 D$-model, the perturbations amplitude $a$ (cf. Eq. (3)) ranges from $\sqrt{3} \cdot 10^{-9}$ to $\sqrt{3} \cdot 10^{-3}$.

the one hand the dispersion is small for the large explored range of perturbation amplitudes, on the other hand $2 D$ and $3 D$ simulations give close results.

In conclusion, these results complete and confirm previous analyses concerning the onset of convection for the thermal transient regime [11,12]. Stability analysis is meaningful for this regime, in the sense that it is possible to characterize a thin transition region in the control parameters space between stable and unstable configurations. This region is not very sensitive to disturbance description (shape and amplitude) and is similar in $2 D$ and $3 D$ models.

At last, some simulations have been performed on $3 D$ configurations of smaller aspect ratio. Thresholds are not very different, since a relative difference of less than $11 \%$ is obtained when reducing the aspect ratio from $A=10$ to $A=3$ and, of less than $43 \%$, for a reduction from $A=10$ to $A=1$.

\subsection{Convective patterns}

Unlike $2 D$ simulations, the $3 D$ configuration allows to describe more accurately the convective patterns and their time evolutions. Fig. 5 and Fig. 6 show the temperature field evolutions at the free surface for two configurations, $e=1 \mathrm{~mm}, \mu=3 \mathrm{mPa} . \mathrm{s}$ and $e=30 \mathrm{~mm}, \mu=3500 \mathrm{mPa} . \mathrm{s}$ respectively. Flow patterns are different for these two configurations. Indeed for small thickness corresponding to surface tension driven flow, cells are observed as in classical Rayleigh-BénardMarangoni studies [17]. They grow in time but become also less active, since $P e$ and $\Delta \Theta_{\max }$ 
decrease for $t>1$ (cf. Fig. 2 and Fig. 5). Additional patterns emerge for the largest thickness linked to buoyancy driven flow. The convective flow organizes itself into rolls (Fig. 6) [10].

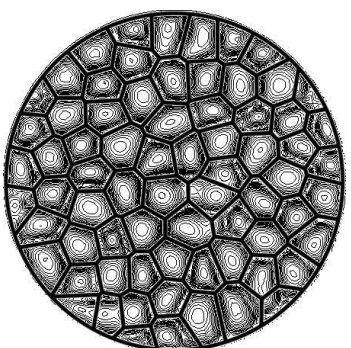

(a) $t=0.7, \mathrm{Pe}=22.95$ $\Delta \Theta_{\max }=12 \times 10^{-2}$ $\Delta \Theta_{\mathrm{fs}}=7.44 \times 10^{-2}$

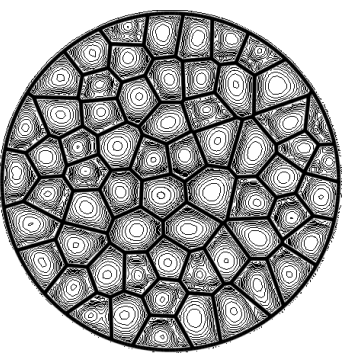

(b) $t=1, \mathrm{Pe}=20.33$, $\Delta \Theta_{\max }=13.2 \times 10^{-2}$ $\Delta \Theta_{\mathrm{fs}}=8.73 \times 10^{-2}$

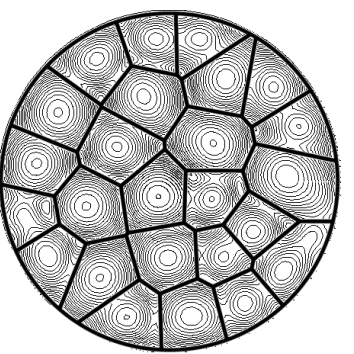

(c) $t=6, \mathrm{Pe}=7.77$, $\Delta \Theta_{\max }=6.26 \times 10^{-2}$ $\Delta \Theta_{\mathrm{fs}}=3.55 \times 10^{-2}$

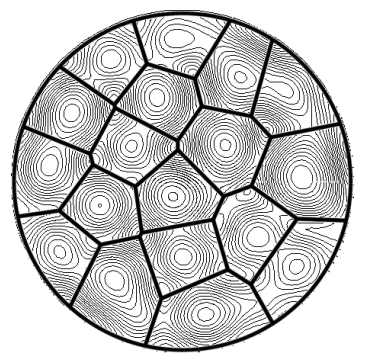

(d) $t=8, \mathrm{Pe}=3.47$, $\Delta \Theta_{\max }=3.57 \times 10^{-2}$, $\Delta \Theta_{\mathrm{fs}}=1.56 \times 10^{-2}$

Fig. 5. Iso-temperature levels and reconstructed cells at the interface, for several successive times, $e=1 \mathrm{~mm}, \mu=3 \mathrm{mPa}$.s. The Peclet number Pe (cf. Fig. 2(b)), the maxima of temperature differences in the fluid layer $\Delta \Theta_{\max }$ (cf. Fig. $\left.2(\mathrm{a})\right)$ and at the free surface $\Delta \Theta_{\mathrm{fs}}$ are specified.

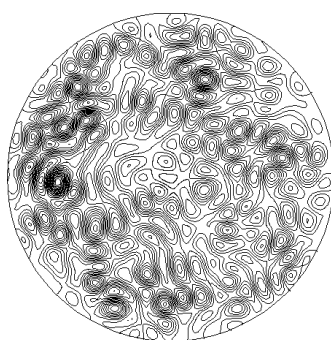

(a) $t=0.2, \mathrm{Pe}=0.486$ $\Delta \Theta_{\max }=0.66, \Delta \Theta_{\mathrm{fs}}=$ $4.7 \times 10^{-3}$

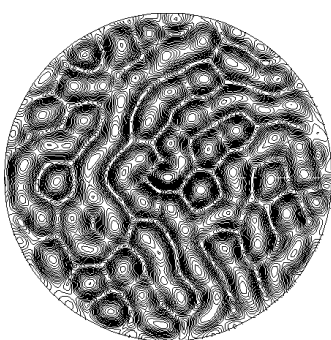

(b) $t=0.5, \mathrm{Pe}=$ $14.04, \Delta \Theta_{\max }=0.473$ $\Delta \Theta_{\mathrm{fs}}=17.9 \times 10^{-2}$

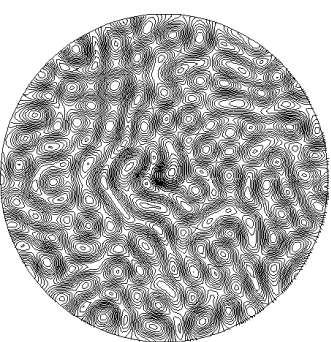

(c) $t=0.8, \mathrm{Pe}=$ $6.62, \Delta \Theta_{\max }=0.273$ $\Delta \Theta_{\mathrm{fs}}=6.83 \times 10^{-2}$

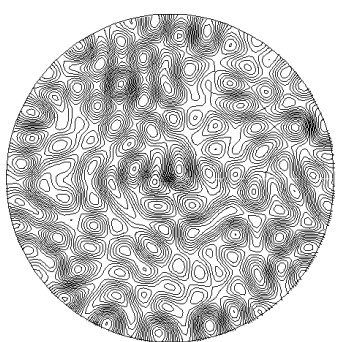

(d) $t=1.1, \mathrm{Pe}=$ 2.3, $\Delta \Theta_{\max }=0.146$ $\Delta \Theta_{\mathrm{fs}}=2.23 \times 10^{-2}$

Fig. 6. Iso-temperaturelevels at the interface, for several successive times, $e=30 \mathrm{~mm}, \mu=3500 \mathrm{mPa}$.s. The Peclet number Pe and maxima of temperature differences in the fluid layer $\Delta \Theta_{\max }$ and at the free surface $\Delta \Theta_{\mathrm{fs}}$ are specified.

To get a more systematic characterization of capillary cells for $e=1 \mathrm{~mm}$ and $\mu=3 \mathrm{mPa.s}$, a reconstruction method has been developed. First, the positions the temperature maxima on the free surface are detected. Then the cell faces are defined as belonging to perpendicular bisectors between the nearest adjacent hot points. This analysis is performed with a Voronoi algorithm (cf. Fig. 5). Fig. 7 presents the evolution of the mean value of the cells size with its standard error. The mean cell size increases by a factor of about two while, as said above, convection fades away (Fig. 2(b)). For purpose of comparison, $2 D$ results, for the same mean initial thermal energy, are also reported. The mean $2 D$ wavelengths, $\overline{\lambda_{2}}$, are defined by the couples of contra-rotating convective cells $\left(\overline{\lambda_{2 D}}=(2 A) /(2 n)\right.$ with $2 A$ the cavity extension and $n$ the number of cells). For $t>0.5$, the mean sizes of $3 D$-capillary cells at the free surface are in good agreement with the $2 D$ patterns. The $2 D$ model seems to overestimate the wavelength of about $30 \%$ at the beginning of the drying. However, it is important to recall that the flow is then almost conductive and it is thus very difficult to characterize the cell size.

To go a little further in the characterization of the cell shapes and their time evolution during the transient thermal regime, cells coming from 100 different shots are classified with 




Fig. 7. Dimensionless cell size: mean values and standard errors

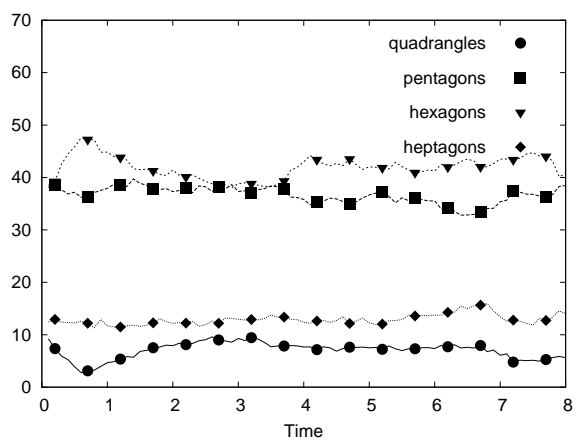

Fig. 8. Percentage of quadrangles, pentagons, hexagons and heptagons

respect to the number of edges as follows. For each cell, only edges greater than 0.1 times the largest side are considered. Cells in contact with the boundary are not taken into account. It can be seen in Fig. 8 that the majority of the patterns are pentagons and hexagons and the proportions do not evolve noticeably. The hexagonal shape is very usual in the classical BénardMarangoni configuration, provided that the aspect ratio of the liquid layer is large enough and the cells are far from lateral walls. Notice that pentagons and squares have also been observed in numerical and experimental studies in case of square containers [17].

The present categorization of flow structures is only based on geometrical considerations but the observed patterns can differ substantially from their ideal shapes (edges of same length) as obtained in the steady Bénard-Marangoni problem [17]. To measure the departure of each cell from its regular polygon shape, the following procedure is used. For a hexagonal cell for instance, we measure its area and define the reference regular hexagon having the same area. The cell is divided up into six triangles with the cell center as apex and the six cell edges as base of the triangles. Then the standard error $\sigma$ between the area of the reference triangle $(1 / 6$ of the hexagon area) and the six triangles of the cell is computed and used to characterize the cell regularity. Results are given in Fig. 9. The percentage of cells having a standard error in

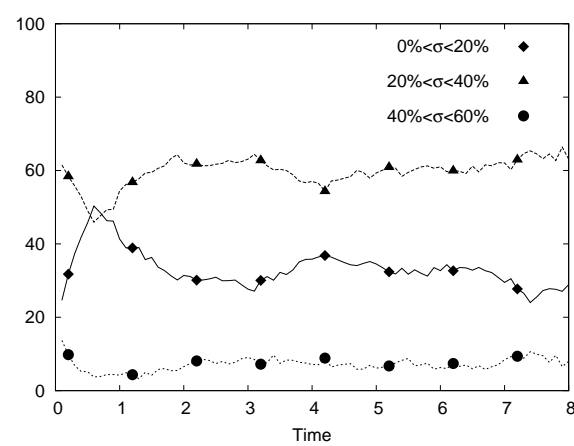

(a) Pentagonal cells

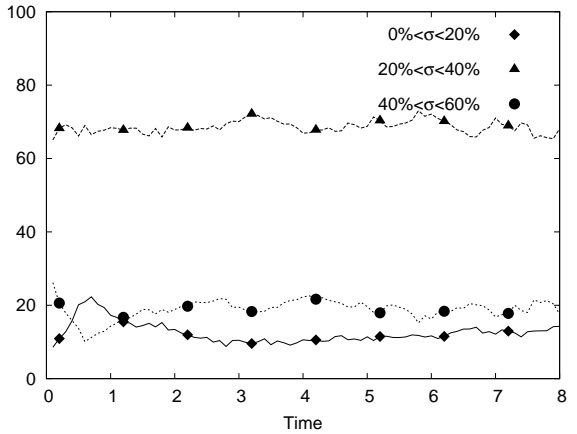

(b) Hexagonal cells

Fig. 9. Evolution of the regularity measured by standard error with respect to regular pentagons or hexagons. $e=1 \mathrm{~mm}, \mu=3 \mathrm{mPa}$.s. 
the range $[20 \% ; 40 \%]$ is about $60 \%$ all along the evolution of the transient regime. The cells induced by evaporation with random initial conditions are less regular than those observed in the usual steady Bénard-Marangoni configuration. Notice however that, more regular cells are recovered if initial conditions are smooth or well structured in space, but this necessitates a very accurate control of the initial condition, what is really hard to achieve in experimental setups.

\section{Conclusion}

The thermal transient regime, occurring at the beginning of the drying of a polymer/solvent solution, has been studied numerically. A particular attention has been payd to the impact of the initial conditions by means of several stochastic models of perturbations. The main result is that it is possible to characterize a thin transition region between stable and unstable configurations and this region is not very sensitive to the shape and amplitude of the disturbance. The onset of convection has been determined, with $2 D$ and $3 D$ models, for a large range of initial thicknesses and viscosities. The results complete and confirm previous $2 D$ stability analysis. $2 D$ and $3 D$ thresholds are not very different. The $3 D$ model has also been used to characterize the flow pattern evolution during the drying. In the case of convection driven by surface tension, tools have been developed in order to accurately describe the cell morphology. It is shown that cells are mostly non-regular pentagons or hexagons. The cell size increases during the drying while convection fades away.

The next step of this study will concern the transient solutal convection. This problem is more complex since it implies to take into account both thickness reduction and viscosity increase.

\section{Acknowledgment}

This work has been supported by the "Fédération TMC (Ministère de l'Enseignement supérieur et de la Recherche, France)"

\section{References}

1. J. Pearson, J. Fluid Mech. 4, 489 (1958)

2. F. Busse, Rep. Prog. Phys. 41, 1930 (1978)

3. J. Reichenbach, H. Linde, J. of colloid and interface science 84, 433 (1981)

4. D. Goussis, R. Kelly, Int J. Heat Mass Transfer 33, 2237 (1990)

5. P. Colinet, J.C. Legros, M.G. Velarde, Nonlinear Dynamics of Surface-Tension-Driven Instabilities (Wiley-VCH, 2001)

6. D. Merkt, M. Bestehorn, Physica D 185, 196 (2003)

7. P. Colinet, L. Joannes, C.S. Iorio, B. Haute, M. Bestehorn, G. Lebon, J.C. Legros, Advances in Space Research 32(2), 119 (2003)

8. O. Ozen, R. Narayanan, Phys. Fluids 16, 4644 (2004)

9. C. Moussy, G. Lebon, J. Margerit, Eur. Phys. J. B 40, 327 (2004)

10. G. Toussaint, H. Bodiguel, F. Doumenc, B. Guerrier, C. Allain, Int. J. Heat Mass Transfer 51, 4228 (2008)

11. O. Touazi, E. Chénier, F. Doumenc, B. Guerrier, IJHMT 53, 656 (2010)

12. F. Doumenc, T. Boeck, M. Rossi, B. Guerrier, JFM 648, 521 (2010)

13. O. Bouizi, C. Delcarte, International Journal for Numerical Methods in Fluids 50 (2006)

14. M. Matsumoto, T. Nishimura, ACM Trans. on Modeling and Computer Simulation 8 (1998)

15. Y. Rozanov, Processus aléatoires (MIR, Moscou, 1975)

16. E. Chénier, C. Desceliers, C. Delcarte, B. Trouette, F. Doumenc, B. Guerrier, Sensitivity of diffusive-convective transition to the initial conditions in a transient Bénard-Marangoni problem, in International Heat Transfer Conference, IHTC14 (2010)

17. M. Médale, P. Cerisier, Numerical Heat Transfer, Part A 42, 55 (2002) 